\title{
ABSTRACTS OF THE 7TH INTERNATIONAL CONFERENCE \\ ON ADVANCES IN \\ HEMATOLOGY AND ONCOLOGY \\ (ICAHO2019)
}

\author{
29-30 June, 2019, \\ Issaquah, WA, USA
}

Organized by

The Binaytara Foundation 
1

\section{ASYMPTOMATIC CERVICAL STIFFNESS AS THE SOLE PRESENTING FEATURE OF OVARIAN FOLLICULAR LYMPHOMA: THE VALUE OF HANDS-ON MEDICINE}

\author{
Mahum Shahid ${ }^{1}$, Michael Schroeder ${ }^{1}$, \\ Kathryn Radigan ${ }^{2}$ and Alla O. Zamulko ${ }^{1}$ \\ ${ }^{1}$ University of South Dakota, Sanford \\ School of Medicine, Sioux Falls, SD, U.S.A.; \\ ${ }^{2}$ Sanford Women's Internal Medicine \\ Clinic, Sioux Falls, SD, U.S.A.
}

Background: Breast cancer is one of the most common types of cancer among US women, accounting for $26 \%$ of all newly diagnosed cancer cases in women (1). Follicular lymphoma is a separate clinicopathological entity with tumor derived from germinal center B-cells. Concurrent existence of both types of cancer has been extremely rare with less than ten reported cases in literature (2). Case Report: A 71year-old Caucasian female, with history of invasive ductal carcinoma, status post bilateral mastectomy and completion of 5-year anastrozole therapy, presented for her annual Well Woman examination. An abnormally firm cervix was appreciated on pelvic examination. Subsequent transvaginal ultrasound revealed a thickened endometrium measuring 5.6 $\mathrm{mm}$, as well as a solid left adnexal mass measuring $5.6 \times 5.4 \times 3.9 \mathrm{~cm}$ and the CA-125 level was mildly elevated at $51 \mathrm{U} / \mathrm{ml}$. Computed tomography (CT) of the abdomen and pelvis further showed an enlarged left external iliac chain lymph node and increased density of the mesentery in the lower right quadrant. The patient underwent robotic total laparoscopic hysterectomy with bilateral salpingooophorectomy. Intraoperatively, a $5 \mathrm{~cm}$ solid mass was removed from the left ovary. The morphology and immunohistochemical staining patterns of the ovarian mass was consistent with a diagnosis of follicular lymphoma. Bone marrow biopsy was negative for malignancy but findings on positron-emission tomography/CT were concerning for disease involving cervical and external iliac nodes, left and right submandibular glands, and right parotid gland. The final diagnosis was stage IIIA, grade $3 \mathrm{~A} / \mathrm{B}$ follicular lymphoma. Conclusion: Follicular lymphoma is generally diagnosed at later stages with waxing and waning lymphadenopathy. Ovaries only comprise $0.5 \%$ of extranodal non-Hodgkin's lymphomas $(3,4)$. On the other hand, primary ovarian lymphomas account for $1.5 \%$ of all ovarian neoplasms (4). This atypical presentation of an asymptomatic primary ovarian non-Hodgkin's lymphoma emphasizes the importance of skilled physical examination in the primary care setting, which can help in the early diagnosis of various indolent diseases, including cancer.
1 Jemal A, Siegel R, Ward E, Hao Y, Xu J and Thum MJ: Cancer statistics, 2008. CA Cancer J Clin 58: 71-96, 2008. PMID: 18287387. DOI: 10.3322/CA.2007.0010

2 Tamaoki M, Nio Y, Tsuboi K, Nio M, Tamaoki M and Maruyama R: A rare case of non-invasive ductal carcinoma of the breast coexisting with follicular lymphoma: A case report with a review of the literature. Oncol Lett 7: 10011006, 2014. PMID: 24944658. DOI: 10.3892/ol.2014.1885

3 Nasioudis D, Kampaktsis PN, Frey M, Witkin SS and Holcomb K: Primary lymphoma of the female genital tract: An analysis of 697 cases. Gynecol Oncol 145: 305-309, 2017. PMID: 28284518. DOI: 10.1016/j.ygyno. 2017.02 . 043

4 Dimopoulos MA, Daliani D, Pugh W, Gershenson D, Cabanillas F and Sarris AH: Primary ovarian nonHodgkin's lymphoma: outcome after treatment with combination chemotherapy. Gynecol Oncol 64: 446-450, 1997. PMID: 9062148. DOI: 10.1006/gyno.1996.4583

\section{MULTICENTRIC CASTLEMAN'S DISEASE: A CASE REPORT}

2

Rediet Gebeyehu, Temitope Olatunji-Bello, Aderajew Tadesse and Admasu Tessema

Internal Medicine Residency Program, WellStar Atlanta Medical Center, Atlanta, GA, U.S.A.

Background: Multicentric Castleman's Disease (MCD) is a rarely described proliferative disease of lymph nodes, closely associated with Kaposi's sarcoma (KS) and human immunodeficiency virus (HIV) (1). Our case report describes the systemic manifestations, diagnosis and current treatment modalities of the disease. Case Report: A 25-year-old male with a past medical history of AIDS, hepatitis B and Kaposi's sarcoma presented with 3 weeks of abdominal pain, constipation and night sweats. Physical examination revealed generalized lymphadenopathy, reduced breath sounds and abdominal tenderness. Laboratory studies showed pancytopenia, CT of the abdomen showed bilateral pleural effusions and diffuse intra-abdominal lymphadenopathy. The patient underwent thoracentesis and axillary lymph node excisional biopsy. Elevation in interleukin-6 (IL6), erythrocyte sedimentation rate and C-reactive protein were noted. Oral prednisone transiently improved his symptoms. However, the patient had recurring pleural effusions necessitating repetitive thoracenteses and Pleurex catheter. Pathology revealed KS and MCD with positivity for human herpesvirus-8 (HHV8). The patient was started on chemotherapy with etoposide and rituximab, and antiviral valganciclovir and completed four cycles of chemotherapy. Subsequently, he had acute hypoxemic respiratory failure, 
refractory hypotension, anasarca, disseminated intravascular coagulopathy and multi-organ failure. He later developed cardiorespiratory arrest and died. Discussion: This case provides insight into the rare clinical entity known as MCD. Its incidence is unknown, and etiology is poorly understood (1), however HHV8, present in our patient, has been implicated in $100 \%$ of HIV-infected individuals (2). The disease presents with systemic symptoms such as night sweats, attributed to elevation of IL6, responsible for capillary leaks, leading to pleural effusions (3), as noted in our patient. The current approach to treatment of this disease was used in this case, which includes a combination of IL6directed therapy (rituximab), cytotoxic chemotherapy (etoposide) and HHV8 suppression (valganciclovir) (4). Our patient had a rapidly progressive course that led to mortality within months, corroborating the poor prognosis, with a previously reported 5-year mortality rate of $18 \%$ (1). Nevertheless, a high index of suspicion of MCD in patients with HIV presenting similarly is encouraged in order to allow early diagnosis and treatment.

1 Sarrot-Reynauld F: Castleman's Disease; Orphanet encyclopaedia, August 2001. Available at: http:// www.orpha.net/data/patho/GB/uk-castleman.pdf

2 Soulier J, Grollet L, Oksenhendler E, Cacoub P, CazalsHattem D, Babinet P, d'Agay MF, Clauvel JP, Raphael M, Degos L and Sigaux F: Kaposi's sarcoma-associated herpesvirus-like DNA sequences in multicentric Castleman's disease. Blood 86: 1276-1280, 1995. PMID: 7632932.

3 Dispenzieri A, Armitage JO, Loe MJ, Geyer SM, Allred J, Camoriano JK, Menke DM, Weisenburger DD, Ristow K, Dogan A, Habermann TM: The clinical spectrum of Castleman's disease. Am J Hematol 87(11): 997-1002, 2012. PMID: 22791417. DOI: 10.1002/ajh.23291

4 Chan KL, Lade S, Prince HM and Harrison SJ: Update and new approaches in the treatment of Castleman disease. J Blood Med 7: 145-158, 2016. PMID: 27536166. DOI: 10.2147/JBM.S60514

\section{3}

\section{PROCALCITONIN PERPLEXITY:}

PROLONGED IDIOPATHIC ELEVATION IN UNDIFFERENTIATED PLEOMORPHIC SARCOMA

Thomas Volberding ${ }^{1}$, Mary Dick ${ }^{1}$, Caleb Freeman ${ }^{1}$, Alheli Arce Gastelum ${ }^{2}$, Dinesh Reddy Apala ${ }^{2}$ and Maryam Gbadamosi-Akindele ${ }^{3}$

${ }^{1}$ Creighton University School of

Medicine, Omaha, NE, U.S.A.;

${ }^{2} \mathrm{CHI}$ Health Creighton University

Medical Center, Omaha, NE, U.S.A.;

${ }^{3} \mathrm{CHI}$ Health Creighton University Medical Center

affiliation Veterans Affairs, Omaha, NE, U.S.A.
Background: Historically, elevation in procalcitonin (PCT) has been implicated in medullary thyroid cancer and neuroendocrine tumors. More recently, evaluation of the trend of PCT level has been suggested as useful in monitoring infection, especially in patients with cancer. Its increase serves as a marker of bacterial, fungal, and protozoal infections. During homeostasis, PCT is produced by most tissues in the body at an extremely low level $(<0.01$ $\mathrm{ng} / \mathrm{ml}$ ) and is often induced by bacterial endotoxins. In patients with cancer, additional factors influence its level. Metastasis, in particular, is linked with an elevated PCT level. Case Report: We present the case of an afebrile patient with undifferentiated pleomorphic sarcoma who underwent 25 rounds of radiation therapy and curiously presented 1 month later with elevated procalcitonin level $(5.0 \mathrm{ng} / \mathrm{ml})$, lactic acid of $2.5 \mathrm{mmol} / \mathrm{l}$, and leukocytosis $\left(14.2 \times 10^{3} / \mu \mathrm{l}\right)$. He consistently had a negative workup for infectious agents. Findings were incidental after a hospital visit for dehydration. Leukocytosis and lactic resolved after 4 days into hospitalization. PCT, however, remained elevated more than 4 months. The patient had no findings of metastatic disease. To our knowledge there is no report in the literature describing a prolonged elevation of PCT in a patient with a non-metastatic sarcoma without any signs of infection or any other underlying cause. Discussion: The elevation of PCT has been noted in patients post burn, trauma, minor and major surgery, and cardiogenic shock in addition to infection. Increase has served as a sign of worsening patient outcome and elevated rate of complications. A rise in PCT has also begun to play a part in antibiotic stewardship as it can allow differentiation of autoimmune inflammation from true infectious processes. The use of PCT trend has been increasing in value, making idiopathic elevations found in combination with undifferentiated pleomorphic sarcoma an important addition to the literature.

\section{4 \\ METASTATIC OVARIAN CANCER: IS CHEMOTHERAPY REALLY NEEDED?}

Muhammad Zain Farooq ${ }^{1}$, Sheeba Habeeb Ba Aqeel ${ }^{1}$, Najla Khan ${ }^{1}$, Prasanth Lingamaneni ${ }^{1}$ and Anmol Baranwal ${ }^{2}$

${ }^{1}$ Department of Internal Medicine, John Stroger Hospital of Cook County, Chicago, IL, U.S.A.; ${ }^{2}$ Department of Hematology and Oncology, John Stroger Hospital of Cook County, Chicago, IL, U.S.A.

Background: Solitary brain metastasis is rarely seen with ovarian cancer and only a few case reports/series have been published in the literature. We report a case of solitary brain metastasis in a patient with ovarian cancer. Case Report: A 46-year-old female patient presented with complaints of abdominal pain and was found to have serous papillary 
adenocarcinoma with peritoneal carcinomatosis. The patient had three cycles of neoadjuvant chemotherapy with carboplatin/paclitaxel, followed by bilateral oophorectomy and omentectomy. Tumor was optimally debulked. She completed three more cycles of adjuvant carboplatin/ paclitaxel. Five years after completion of chemotherapy with no residual disease, the patient presented with severe headaches. Computed tomography of the head reveled a $3.9 \times 3.0 \times 3.3 \mathrm{~cm}$ mass in the right cingulate gyrus. The patient underwent right frontal craniotomy with open biopsy of right subcortical brain tumor followed by debulking of the tumor. Brain biopsy was consistent with metastatic carcinoma from the ovaries. The patient then underwent whole-brain radiation therapy, $30 \mathrm{~Gy}$ in 10 fractions. After discussion of the risks and benefits of chemotherapy, our patient chose to continue with active surveillance only. Repeat computed tomographic scans have continued to demonstrate decreasing size of the brain lesion with no evidence of any recurrence of malignancy. Conclusion: Brain metastasis from ovarian cancer has a poor outcome, with median overall survival of 8.2 months. Due to its rarity, there are no consistent guidelines on management of a solitary brain metastasis from ovarian cancer. In literature, patients have mostly received multi-modal treatment with surgery, chemotherapy and radiation therapy. Our patient had a solitary brain metastasis with no evidence of worsening metastasis after resection and radiation therapy. Our case demonstrates that patients with metastasis can be managed without exposing them to the harmful effects of chemotherapy.

\section{5}

\section{A CASE OF DOUBLE HIT LYMPHOMA IN HIV}

Sheeba Habeeb Ba Aqeel, Muhammed Zain Farooq and Vatsala Katiyar

John H. Stroger Hospital of Cook

County, Chicago, IL, U.S.A.

Background: Double-hit lymphoma involving translocations of $M Y C$ and BCL2 or BCL6 gene occur in $<10 \%$ of cases of diffuse large B-cell lymphoma (DLBCL). However, doublehit DLBCL is a rare presentation in HIV-infected individuals that carries poor prognosis if not promptly recognized. Case Report: We report the case of a 39-year-old man with HIV who presented with headache, nausea, vomiting, photophobia and double vision. Physical examination was remarkable only for diplopia. Computed tomography (CT) revealed a $2.5 \mathrm{~cm}$ soft-tissue mass within the clivus with extension into the prepontine cistern and sphenoid sinus. There was no metastasis observed on CT imaging of the chest, abdomen and pelvis. Biopsy of the mass was significant for DLBCL. The patient was promptly started on a first cycle of chemotherapy with rituximab, cyclophosphamide, doxorubicin, vincristine, and prednisone (R-CHOP). However, follow-up CT scan showed unimpressive response in the size of the tumor. Fluorescence in situ hybridization was positive for a double hit involving translocation of MYC and BCL2. Chemotherapy was subsequently changed to cyclophosphamide, vincristine, doxorubicin, high-dose methotrexate/Ifosfamide, etoposide, and high-dose cytarabine (CODOX-M/IVAC). Upon completion of the first cycle, the patient reported resolution of headaches, as well as of double vision, nausea, and vomiting. Repeat CT scan showed interval improvement of the tumor mass. Conclusion: Double-hit lymphoma is extremely rare in HIV-affected individuals and has a propensity to infiltrate the bone marrow or CNS. As in our case, it typically does not respond to standard R-CHOP chemotherapy. Recently, RCODOX-M/IVAC has been shown to be beneficial in patients with HIV as seen in a case like ours. Given the poor prognosis and lack of effective data in terms of prospective trials for double-hit DLBCL in patients with HIV, there is a need for early molecular risk stratification for proper chemotherapeutic regimen of these patients.

\section{6 \\ RISK OF CARDIAC TOXICITY FROM ANTHRACYCLINES AND TRASTUZUMAB PATIENTS AGED 50 YEARS OR MORE WITH BREAST CANCER: A SYSTEMATIC REVIEW AND META-ANALYSIS}

Muhammad Zain Farooq ${ }^{1}$, Muhammad Saad Farooq ${ }^{2}$, V. V. Pavan Kedar Mukthinuthalapat ${ }^{1}$, Sheeba Habeeb Ba Aqee ${ }^{1}$, Prasanth Lingamaneni ${ }^{1}$, Nisar Asmi ${ }^{1}$ and Muhammad Ebad ur Rehman ${ }^{3}$

${ }^{1}$ John Stroger Hospital of Cook

County, Chicago, IL, U.S.A.;

${ }^{2}$ Dow University of Health Sciences, Karachi, Pakistan;

${ }^{3}$ Western Michigan University School of

Medicine, Kalamazoo, MI, U.S.A.

Background: Elderly patients are at increased risk of development of cardiovascular diseases and some studies have shown that they are also at higher risk of cardiac toxicity from anthracyclines and trastuzumab. In this context, we performed a systematic review and meta-analysis to assess the available evidence and accurately determine the risk of increased age for development of this toxicity, respectively. Materials and Methods: PubMed, EMBASE and SCOPUS databases were searched from inception until $2 / 5 / 19$ to identify all articles that studied cardiac toxicity with trastuzumab and anthracyclines in patients with breast cancer. We defined elderly as age greater than 50 years and included observational studies and clinical trials with agebased sub group comparisons. Newcastle Ottawa Scale and 
Cochrane Collaboration tools were used to assess the bias in these studies. Odds ratios comparing cardiac toxicity rates of elderly patients to those younger than 50 years were calculated for each study and pooled estimate of odds ratios were derived using Dersimonian-Laird random effects model. I2 statistic was used to assess heterogeneity, and publication bias was estimated using Egger's regression coefficient. Results: Out of 708 articles retrieved in the initial search, 13 observational studies and clinical trials $(n=26,004$; elderly age patients $=16,544)$ were included in our quantitative analysis. There was low risk of bias in the studies included and there was no evidence of publication bias ( $p$-value $>0.1$ ). Cardiac toxicity was observed in 881 patients and cumulative odds ratio for cardiac toxicity in older patients was 1.5 (95\% confidence interval=1.1-2.1, $\left.p<0.001 ; \mathrm{I}^{2}=74.5 \%, p<0.001\right)$. Conclusion: Our findings suggest that elderly patients are at higher risk of cardiac toxicity with anthracyclines and trastuzumab. A risk-benefit analysis should be undertaken in older patients before administering these agents.

\section{7}

\section{AN UNUSUAL CASE OF DIFFUSE LARGE B-CELL NON-HODGKIN'S LYMPHOMA ERODING THROUGH THE SKULL}

Sharanpreet Kaur ${ }^{1}$, Harrison Chen ${ }^{2}$, Arthur Omuro ${ }^{1}$ and Irene May-Ling Hutchins ${ }^{1}$

${ }^{1}$ Desert Regional Medical Center, Palm Springs, CA, U.S.A.;

${ }^{2}$ Western University of Health Sciences, Pomona, CA, U.S.A.

Background: Diffuse large B-cell lymphoma (DLBCL) is a subtype of non-Hodgkin's lymphoma that can arise from virtually any part of the body. Based on the nature of lymphoma progression, the majority of these tumors develop within lymph nodes, while in contrast, about $30 \%$ of cases present as extranodal disease due to primary or secondary manifestation. Herein, we present an unusual case of DLBCL presenting as a primary extracranial lymphoma eroding through the skull to cause seizures. Case Report: A 64-yearold man with past medical history of hypertension presented to our hospital with seizures and acute left hemiparesis. Immediate non-contrast computed tomography (CT) of the head demonstrated a large extracranial hyperdense mass with calvarial erosion. As the radiographic appearance on followup magnetic resonance imaging of the brain was highly suggestive of atypical meningioma and the patient experienced neurological deterioration with recurrent seizures, he underwent urgent craniotomy and resection of the mass. Final pathology assessment confirmed nonHodgkin's malignant lymphoma, DLBCL. Hematology/
Oncology was consulted and the patient was started on rituximab-cyclophosphamide, adriamycin, vincristine and prednisone (R-CHOP) alternating with intravenous methotrexate. After 3 weeks of rehabilitation, the patient returned to his home state for continued chemotherapy and consideration of consolidative radiation. Discussion: DLBCL most commonly originates in the gastrointestinal tract, but based on lymphatic distributions, other organs and tissues may also be involved such as the testes, central nervous system, and bone. Compared to other extranodal lymphomas, however, primary cranial vault lymphoma remains extremely rare and constitutes only about $0.2 \%$ of all lymphoma cases. This case is unique because the primary lymphoma did not emerge from a lymph node, but rather as a skull mass that eroded through the cranium, extending both extracranially and intracranially. After surgical mass resection, we followed the standard chemotherapy regimen for DLBCL, consisting of R-CHOP alternating with intravenous methotrexate, to which the patient responded very well.

\section{8}

\section{CHECK POINT INHIBITOR-INDUCED FANCONI SYNDROME}

\author{
Saira Farid ${ }^{1}$, Hira Latif $^{1}$ and Chul Kim ${ }^{1,2}$ \\ ${ }^{1}$ MedStar Washington Hospital Center/Georgetown \\ University Hospital, Washington, DC, U.S.A.; \\ ${ }^{2}$ Georgetown University Hospital, Washington, DC, U.S.A.
}

Background: Immune checkpoint inhibitors (ICI) have been approved by the FDA for use in several solid tumor types and hematological malignancies. Immune-related (IR) toxicities are a well-known potential adverse event (AE) of these drugs. Renal toxicity includes acute interstitial nephritis, minimal change disease, and immune complex glomerulonephritis. We present a case of nivolumab/ipilimumab-induced Fanconi syndrome; this is the first reported case in literature to the best of our knowledge. Case Report: A 58-year-old male was diagnosed with extensive-stage small-cell lung cancer with adrenal metastasis. He completed six cycles of cisplatin/etoposide, followed by thoracic and prophylactic cranial irradiation. His disease progressed in the adrenals and brain. He was started on nivolumab, ipilimumab along with CyberKnife radiation. Three weeks into the therapy, the patient developed abdominal pain with grade 3 transaminitis, and required steroids and mycophenolate for presumed IR hepatitis. He subsequently presented to the Emergency Department with worsening abdominal pain. Initial laboratory tests revealed a white blood cell count of $17 \times 10^{3} / \mu \mathrm{l}$, aspartate aminotransferase/alanine aminotransferase 99/210 U/1, direct bilirubin $2.8 \mathrm{mg} / \mathrm{dl}$, blood urea nitrogen of $43 \mathrm{mg} / \mathrm{dl}$, creatinine of $2.31 \mathrm{mg} / \mathrm{dl}$ (baseline: $1.3 \mathrm{mg} / \mathrm{dl}$ ), phosphorus of $2.3 \mathrm{mg} / \mathrm{dl}$, and glucose of $303 \mathrm{mg} / \mathrm{dl}$ with metabolic acidosis. 
He had a negative viral hepatitis panel, and there was no hydronephrosis on renal ultrasound. Urinalysis showed glucosuria $(>500 \mathrm{mg} / \mathrm{dl})$ and proteinuria $(30 \mathrm{mg} / \mathrm{dl})$ with FeNa of $2 \%$. This gave rise to concerns for FS with proximal tubulopathy. He was started on intravenous bicarbonate, methylprednisolone and vancomycin/zosyn. Urinary phosphorus and uric acid were both elevated at $22.1 \mathrm{mg} / \mathrm{dl}$ and $37.7 \mathrm{mg} / \mathrm{dl}$, respectively. FS/proximal tubular damage secondary to immunotherapy was diagnosed. He was discharged on oral bicarbonate and steroid taper; his creatinine level returned to baseline at 3 weeks. The patient was started on topotecan, but had disease progression and decided on comfort care. Conclusion: Increased ICI use should warrant attention to adverse events including renal complications. Renal monitoring is vital for early detection of renal adverse events and prevention of severe renal damage. Early initiation of steroids and supportive therapy can result in favorable outcomes.

\section{9}

\section{A CASE OF ACUTE KIDNEY INJURY ASSOCIATED WITH TRAMETANIB/DABRAFENIB}

\author{
Lauren Ogawa ${ }^{1,2}$ and Nishkarsh Saxena ${ }^{2,3}$ \\ ${ }^{1}$ Midwestern University, Glendale, AZ, U.S.A.; \\ ${ }^{2}$ Northern Arizona Healthcare, Cottonwood, AZ, U.S.A.; \\ ${ }^{3}$ Southwest Kidney Institute, Cottonwood, AZ, U.S.A.
}

Background: BRAF inhibitor, dabrafenib, interrupts cell signaling and slows cell growth, providing a targeted treatment for $40-50 \%$ of melanoma cases with $B R A F$ mutation, but has been rarely associated with nephrotoxicity (1). We present a case of acute kidney injury (AKI) in association with tramatenib/dabrafinib therapy. Case Report: A 60-year-old diabetic female with advanced melanoma on therapy with trametanib/dabrafenib, presented with nausea, vomiting and watery diarrhea of 1 week's duration. At the Emergency Department, blood pressure was 79/41 mmHg. Examination revealed dry oral mucosa. Significant laboratory results included serum creatinine $(\mathrm{Cr})$ of $1.59 \mathrm{mg} / \mathrm{dl}$ (baseline: $0.8 \mathrm{mg} / \mathrm{dl}$ ), alanine aminotrasferase $56 \mathrm{U} / \mathrm{l}$, aspartate aminotransferase $134 \mathrm{U} / 1$ and platelets of $79 \times 10^{3}$ cells $/ \mu 1$. Renal ultrasound was unremarkable. Admitted for hypovolemic shock and acute kidney injury (AKI) secondary to gastrointestinal losses and dehydration, she received intravenous fluids along with vancomycin and piperacillintazobactam. Infectious work-up was negative. Despite fluids and discontinuation of antibiotics, kidney function deteriorated, with serum $\mathrm{Cr}$ reaching $3.51 \mathrm{mg} / \mathrm{dl}$. Serological studies including antineutrophil cytoplasmic antibodies, antinuclear antibodies, double-stranded DNA antibodies, serum complements, and hepatitis B/C panel were normal. Renal biopsy revealed acute tubular necrosis and patchy tubulointerstitial nephritis. She received prednisone $1 \mathrm{mg} / \mathrm{kg}$ for four weeks followed by taper and BRAF inhibitor therapy was held. Subsequently, serum Cr improved to $1.3 \mathrm{mg} / \mathrm{dl}$ by the end of steroid therapy. Discussion: AKI was likely due to chemotherapy, complicated by gastrointestinal losses from gastroenteritis. The patients also had elevated liver enzyme levels and thrombocytopenia, which are associated with use of BRAF inhibitors (2). Nephrotoxicity is a rare complication of dabrafenib therapy, but reports suggest a dose-dependent association with development of AKI, with renal biopsies showing two main patterns of injury: Acute tubular necrosis and acute interstitial nephritis (1, 3). Conclusion: Early detection and treatment of AKI associated with BRAF therapy can prevent irreversible renal injury, making it important to monitor kidney function closely in patients treated with trametanib/dabrafenib.

1 Wanchoo R, Jhaveri KD, Deray G and Launay-Vacher V: Renal effects of BRAF inhibitors: A systematic review by the Cancer and the Kidney International Network. Clin Kidney J 9(2): 245-251, 2016. PMID: 26985376. DOI: $10.1093 / \mathrm{ckj} / \mathrm{sfv} 149$

2 Welsh SJ and Corrie PG: Management of BRAF and MEK inhibitor toxicities in patients with metastatic melanoma. Ther Adv Med Oncol 7(2): 122-136, 2015. PMID: 25755684. DOI: $10.1177 / 1758834014566428$

3 Ikesue H, Nagano T and Hashida T: A case of acute kidney injury associated with dabrafenib and trametinib treatment for metastatic melanoma. Ann Pharmacother 52(10): 10511052, 2018. PMID: 29890845. DOI: 10.1177/10600280 18782985

\section{0 \\ UTILIZATION OF 4T PRETEST PROBABILITY SCORING SYSTEM IN HEPARIN-INDUCED THROMBOCYTOPENIA AT WELLSTAR ATLANTA MEDICAL CENTER: A QUALITY IMPROVEMENT STUDY}

Rediet Gebeyehu, Aderajew Tadesse, Getnet Tioum, Douglass Collins and Admasu Taddesse

Wellstar Atlanta Medical Center, Atlanta, GA, U.S.A.

Background: Heparin-induced thrombocytopenia (HIT) is a life-threatening complication of heparin therapy, leading to extensive thrombosis. It occurs in $5 \%$ of patients exposed regardless of dose, schedule or route of administration. Diagnosis of HIT depends on clinical suspicion determined by $4 \mathrm{~T}$ probability scoring system and laboratory testing with immunoassays that detect HIT anti-platelet factor 4 antibodies that have a sensitivity of $96-100 \%$ but a specificity of $68-94 \%$. Serotonin-release assay is a gold standard but expensive form of testing, with sensitivity and 
specificity $>96 \%$. The primary purpose of this study was to assess proper utilization of $4 \mathrm{~T}$ score as a pretest clinical diagnosis tool at the WellStar Atlanta Medical center in Atlanta, GA, USA. Materials and Methods: A retrospective electronic chart review of patients who were admitted to WellStar Atlanta Medical center between, from October 2013 and October 2015 and had HIT testing done between October 2013 and October 2015. Those who had baseline thrombocytopenia $(<100,000 / \mu 1)$ at the time of admission and patients with known hematological malignancies or any platelet-related pathologies were excluded from the study. After calculating the $4 \mathrm{~T}$ score retrospectively, we identified the proportion of patients who had low probability on $4 \mathrm{~T}$ score testing and yet had unnecessary antibody testing and those with intermediate and high probability who had proper testing. Results: Review of 134 patients (average age $=63.6$ years), showed $50.7 \%$ of the patients had a low probability on 4T scoring system and had unnecessary testing. Antibody testing was appropriate in the remaining patients, who had intermediate and high probability testing. There were $15.7 \%$ of patients who had never had heparin exposure, but yet underwent antibody testing. Conclusion: This quality improvement study showed a widespread improper utilization of $4 \mathrm{~T}$ probability testing at our hospital and the related unnecessary healthcare cost. Recommendations were made to the hospital administration to incorporate 4T probability score calculator in the electronic medical record system to easily identify patients needing further testing.

\section{1}

\section{UTILITY OF ENDOSCOPIC ULTRASOUND IN THE WORK UP FOR SOLITARY HEPATOBILIARY NEUROENDOCRINE LESIONS}

Moataz Ellithi, Muhammad Danial Siddiqui and Muslim Atiq

University of South Dakota Sanford School of Medicine, Sioux Falls, SD, U.S.A.

Background: Primary neuroendocrine tumors of the liver and biliary tract are extremely rare as the vast majority of neuroendocrine tumors are the result of metastasis from the gastrointestinal tract or lungs. Herein, we present a case of hepatobiliary neuroendocrine tumor that was later found to have an extrahepatic lesion on endoscopic ultrasound (EUS), which was not detected initially on imaging, radioisotope scanning, or intraoperative ultrasound. Case Report: A 43year-old woman, with an unremarkable past medical history, presented with a 3-week history of generalized itching and abdominal pain. She had jaundice and right upper quadrant tenderness on examination. Initial workup showed mild direct hyperbilirubinemia with a mildly elevated alkaline phosphatase with normal aspartate transaminase and alanine transaminase levels. Initial imaging showed an amorphous, regionally invasive, and obstructing soft-tissue mass in the region of the hepatic hilum that involved both the bile ducts and portions of the left hepatic lobe. The middle third of the main bile duct was found to harbor a polypoid mass on endoscopic retrograde cholangiopancreaticography. A biliary stent was placed after biopsies were obtained. Biopsy revealed nests of neoplastic cells that were subsequently identified as well-differentiated neuroendocrine tumor. A search for a possible primary neuroendocrine tumor was performed and included imaging of the chest, abdomen, and pelvis, a colonoscopy, capsule endoscopy, and octreotide scan, however, no primary tumor outside of the liver was identified. Surgical debulking was performed, during which intraoperative exploration and ultrasound failed to reveal any extrahepatic tumor sanctuaries. A few months later, due to recurrent abdominal pain, the patient underwent EUS, which revealed a lesion in the pancreas. It was unclear, however, whether it was a primary or metastatic lesion. Conclusion: This case represents a diagnostic challenge and emphasizes the potential utility of EUS in the preoperative work up for any presumable primary hepatobiliary neuroendocrine tumor.

\section{2 \\ ANAPLASTIC MULTIPLE MYELOMA WITH COMPLETE RESPONSE TO BORTEZOMIB-BASED TRIPLET REGIMEN}

\author{
Moataz Ellithi, Hafez Mohammad Abdullah and \\ Mohamed Omar
}

University of South Dakota Sanford School of Medicine, Sioux Falls, SD, U.S.A.

Background: Anaplastic multiple myeloma is a rare and aggressive variant of multiple myeloma that is almost always associated with a very poor prognosis and remains refractory to most treatment regimens. Herein we report a case of anaplastic myeloma with a complete response to bortezomibbased triplet regimen. Case Report: A 52-year-old woman presented with worsening right knee pain. She also reported chronic fatigue with no associated fever, chills, night sweats, or weight loss. She had a medical history of morbid obesity, bilateral knee osteoarthritis, and hypertension. There was no lymphadenopathy or hepatosplenomegaly on physical examination. Initial laboratory tests showed serum calcium of 15.2 (normal range: $8.6-10.3 \mathrm{mg} / \mathrm{dl}$ ), creatinine of 1.8 (normal range $0.6-1.2 \mathrm{mg} / \mathrm{dl}$ ), and hemoglobin of 7.3 (normal range 12-16 g/dl). The peripheral blood smear revealed no circulating blast or plasma cells. Serum protein electrophoresis and serum free light chain assays showed a monoclonal protein spike, IgG Lambda gammopathy, and a kappa/lambda ratio of 0.01 . Bone survey revealed focal areas of osteolytic lucency involving calvaria, distal metaphyses of 
both femurs and distal right humerus diaphysis. Subsequent bone marrow biopsy revealed hypercellular marrow with $80 \%$ involvement by anaplastic plasma cell myeloma lambda chain restricted. Fluorescence in situ hybridization (FISH) analysis revealed $14 \mathrm{q}$ deletion, $3 \mathrm{q}$ gain, $7 \mathrm{p}$ loss, $8 \mathrm{p}$ loss, $8 \mathrm{q}$ gain, $9 \mathrm{p}$ loss, 9p-q gain, monosomy $13,14 q$ micro-deletion, $16 q$ loss, and $18 \mathrm{p}$ gain. These findings were consistent with anaplastic multiple myeloma. She was not considered a candidate for stem cell transplant. Initial treatment with six cycles of velcade, decadrone and lenalidomide (REVLIMID ${ }^{\circledR}$ ) resulted in complete response, with repeat testing showing absence of M-spikes, normalization of $\operatorname{IgM}$ levels, and absence of significant population of plasma cells on flow cytometric immunophenotyping and FISH analysis of a repeat marrow biopsy. Conclusion: Due to the rarity of anaplastic multiple myeloma, no clinical trials are available for those patients with less known about disease prognosis and treatment response.

\section{3 \\ CARDIAC TAMPONADE AND PURULENT PERICARDITIS IN THE SETTING OF AN ESOPHAGEAL PERICARDIAL FISTULA AS AN INITIAL PRESENTATION OF SQUAMOUS CELL CARCINOMA OF THE ESOPHAGUS}

Mahum Shahid ${ }^{1}$, Moataz Ellithi ${ }^{1}$, Ammar Abdullah $^{1}$ and Muhammad Omar ${ }^{2}$

${ }^{1}$ Department of Internal Medicine, University of South Dakota Sanford School of Medicine, Vermillion, SD, U.S.A.;

${ }^{2}$ Avera Mckennan Hospital and University

Health Center, Sioux Falls, SD, U.S.A.

Background: Cardiac tamponade and pericardial effusions have previously been reported in association with esophageal cancer (1). Rarely esophageal pericardial fistulas (EPF) have been reported as the culprits (2). Case Report: We present the case of a 61-year-old female who was found to have an EPF due to esophageal squamous cell cancer that resulted in a purulent pericardial effusion. Results: A 61-year-old female presented with worsening acute-onset severe epigastric and chest pain. Chest computed tomography showed a moderate to large pericardial effusion with a small amount of air in the effusion and the superior mediastinum. There was air projecting outside the lumen of the esophagus at the left atrial level that was concerning for esophageal perforation. An echo showed a large circumferential pericardial effusion and a diastolic collapse of the right ventricle consistent with tamponade physiology. The patient was initially given emergency supportive treatment and was admitted to the ICU. Cardiothoracic surgery performed median sternotomy and drained over 11 of pus from the pericardial space, and placed stents in the pericardium for drainage. Surgeons were able to visualize a $2 \times 2 \mathrm{~cm}$ hole in the back of the pericardium. An endoscopy performed revealed an esophageal perforation with a fistula that connected directly with the pericardial space through a defect in the pericardium posteriorly. An esophageal stent was attempted but was not possible due to the friable esophageal wall. The patient's esophageal biopsy was positive for squamous cell cancer, which later turned out to be stage IIIb after oncological work-up. The patient refused any more aggressive treatments and was discharged to a hospice where she passed away 13 days after presentation. Conclusion: Patients with esophageal cancer and EPF tend to have poor outcome and should be managed with urgent surgical decompression and possible palliative esophageal stenting.

1 Fraser RS, Viloria JB and Wang NS: Cardiac tamponade as a presentation of extracardiac malignancy. Cancer 45(7): 1697-1704, 1980. DOI: 10.1002/1097-0142(19800401) 45:7<1697::AID-CNCR2820450730>3.0.CO;2-J

2 Saluja SS, Puri SK, Arora A, Chander Sharma B and Mishra PK: Esophagopericardial fistula: A delayed complication after esophageal stenting. Am Surg 79(5): $550,2013$.

\section{4}

\section{SUCCESSFUL MANAGEMENT OF HIGH-GRADE PSA-NEGATIVE PROSTATE CANCER WITH IMMUNOTHERAPY}

Mahum Shahid, Radowan Elnair and Christopher Sumey

University of South Dakota Sanford School

of Medicine, Sioux Falls, SD, U.S.A.

Background: High-grade prostate-specific antigen (PSA)negative prostate cancer ( $\mathrm{PCa}$ ) has an increased cancer-related mortality (1). Their anaplastic nature poses a diagnostic dilemma while evaluating site of origin. We present a case of a 78-year-old man with poorly differentiated PSA negative $\mathrm{PCa}$ who was successfully managed with immunotherapy. Case Report: A 78-year-old man presented with recurrent hematuria. Computed tomographic (CT) scan of the abdomen and pelvis revealed thickened bladder wall, enlarged prostate and pelvic lymphadenopathy along with a lytic destructive lesion of the right inferior pubic ramus. CT-guided biopsy of an external iliac lymph node showed poorly differentiated non-small cell carcinoma (NSCC) with immunohistochemistry (IHC) positive for CDX2 and pan-keratin, and negative for PSA, suggestive of upper gastrointestinal or pancreatobiliary tumor. PSA level was normal. Positron-emission tomography/CT scan demonstrated a hyper metabolic mass measuring $7.5 \times 7.3 \mathrm{~cm}$, involving the prostate, rectum and the right inferior pubic bone, with hyper-metabolic pelvic lymph 
nodes. Digital rectal examination revealed a firm mass in the anterior bowel wall with extrinsic stenosis of the rectum. Colonoscopy was unrevealing. Endoscopic ultrasound of the region showed a hypo-echoic heterogenous mass in the anterior peri-rectal space. Fine-needle aspiration cytology again revealed NSCC. A working diagnosis of high-grade PSA-negative $\mathrm{PCa}$ was made based on radiographic and pathology findings. Genomic testing showed tumor mutational burden of greater than 150 mutations/ $\mathrm{Mb}$ and high microsatellite instability but negative programmed death-ligand 1 by IHC. Treatment with pembrolizumab was started. The patient has successfully tolerated 20 cycles, with ongoing response per imaging. Conclusion: High-grade PCa can carry a poor prognosis (2). Single-agent immune checkpoint inhibitors have shown limited utility in PCa. Upfront genomic testing of this patient's tumor facilitated selection of a highly effective first-line therapy that was outside the standard approach for locally advanced PCa and spared toxicity of less effective therapy.

1 Mahal BAV, Aizer AA, Efstathiou JA and Nguyen PL: The association of very low PSA with increased cancer-specific death in men with high-grade prostate cancer. J Clin Oncol 33: 62-62, 2015. DOI: 10.1200/jco.2015.33.7_suppl.62

2 Yang DD, Mahal BA, Muralidhar V, Martin NE, Orio PF, Mouw KW, King MT, Choueiri TK, Trinh Q-D, Hoffman KE, Spratt DE, Feng FY and Nguyen PL: Androgen deprivation therapy and overall survival for Gleason 8 versus Gleason 9-10 prostate cancer. Eur Urol 75: 35-41, 2019. PMID: 30554605. DOI: 10.1016/j.eururo.2018. 08.033

15

DISSEMINATED INTRAVASCULAR COAGULATION AND COOMBS-NEGATIVE HEMOLYTIC ANEMIA CAUSED BY DISSEMINATED CYTOMEGALOVIRUS INFECTION IN AN IMMUNOCOMPROMISED FEMALE SUCCESSFULLY TREATED WITH GANCICLOVIR

Ziauddin Syed and Mohammad Muhsin Chisti

Hematology and Medical Oncology, William Beaumont Hospital, Royal Oak, MI, U.S.A.

Background: Disseminated intravascular coagulation (DIC) and hemolytic anemia are rare complications of cytomegalovirus (CMV) infection. They have been observed separately in both immunocompromised and immunocompetent patients in conjunction with CMV infection, however, they have very rarely been observed together. There is limited information regarding the best approach in management of both phenomena which can be life-threatening. Case Report: A 64-year-old Caucasian female with history of end-stage renal disease status post renal re-transplantation on chronic immunosuppressive therapy with mycophenolate, tacrolimus, and prednisone presented with epigastric abdominal pain. She proceeded to demonstrate acute encephalopathy and underwent neurological evaluation, including a lumbar puncture which was positive for CMV by polymerase chain reaction. Further CMV quantitation detected viral loads as high as 25,842,190 IU/ml. Her encephalopathy was attributed to disseminated reactivation of latent $\mathrm{CMV}$ infection as serology was positive for $\mathrm{CMV} \mathrm{IgG}$ in the past. She presented mild thrombocytopenia which continued to worsen. Further workup confirmed DIC with elevated prothrombin time, low fibrinogen level, and elevated D-dimer level. Workup also revealed Coombs-negative hemolytic anemia with schistocytes, low haptoglobin level, and elevated LDH, indirect bilirubin and plasma hemoglobin levels. The patient was started on ganciclovir after mycophenolate and tacrolimus were discontinued. She was transfused with platelets in addition to receiving fresh-frozen plasma and cryoprecipitate. The viral load consistently decreased following treatment and DIC resolved. The patient required only 1 unit of packed red blood cells for hemolytic anemia, with hemoglobin subsequently being stable $>8 \mathrm{~g} / \mathrm{dl}$. However, the level of haptoglobin remained low and $\mathrm{LDH}$ continued to be elevated, indicating persistent hemolysis despite improvement. The patient returned to baseline mentation and she was discharged home on valganciclovir, and immunosuppressive therapy was resumed. Conclusion: In appropriate settings, physicians must consider DIC and non-immune hemolytic anemia in patients with CMV infection.

\section{6}

ROLE OF STEREOTACTIC BODY RADIOTHERAPY IN EARLY-STAGE PANCREATIC CANCER

Amir Bista, Andrew J. Borgert, Dipesh Uprety, Yazhini Vallatharasu, Lubina Arjyal, Deval Rajyaguru, Peter James Polewski, Mamatha Gaddam, Swapna Narayana and Leah L. Dietrich

Gundersen Health System, La Crosse, WI, U.S.A.

Background: Stereotactic body radiotherapy (SBRT) can precisely deliver high doses of radiation to tumors of small volume. It could potentially be considered as an alternative therapy to surgery for patients with early-stage pancreatic cancer who are not surgical candidates. Because of limited literature regarding its use in early-stage pancreatic cancer, we aimed to evaluate the effectiveness of SBRT using the National Cancer Database (NCDB). Patients and Methods: Patients with stage I (T1 or T2, N0, M0) pancreatic adenocarcinoma diagnosed from 2004-2014 were included and those who underwent surgery were excluded. Patients were stratified into two groups: SBRT with or without chemotherapy, and chemotherapy only (CTx). Kaplan-Meier 
analysis with log-rank test and Cox proportional hazards were used to compare the overall survival (OS). Patients were matched on propensity score to account for treatment selection bias. Statistical analyses were conducted utilizing SAS version 9.4. Results: NCDB included a total of 390 patients (71 in the SBRT group and 319 in the CTx group). Eighteen $(25.35 \%)$ patients in the SBRT group received chemotherapy. The majority of patients in the SBRT group were aged 80 years $(61.97 \%)$ or more, Caucasians $(83.1 \%)$, diagnosed in 2009 to 2014 (73.24\%), from a metropolitan area $(81.7 \%)$, with Charlson-Deyo comorbidity score of 0 $(77.5 \%)$ and treated in comprehensive community cancer programs or academic/research programs $(77.5 \%)$. Of the total 173 patients included in propensity-matched analysis (63 SBRT and 110 CTx group), 22 (12.7\%) refused surgery and $31(17.9 \%)$ had contraindication for surgery. On propensity-matched analysis, patients in the SBRT group were found to have significantly better OS compared to the Ctx only group (median OS of 15.7 vs. 10.81 months respectively, $p=0.02$ ). Conclusion: Our study showed significant survival benefit with SBRT over chemotherapy alone in patients with stage I pancreatic cancer who did not receive surgery. SBRT should therefore be strongly considered for this patient population. 